\title{
Fuzzy PID Controller to control the attitude of Quadrotor UAV
}

\author{
DING LI \\ Liding900712@126.com \\ Assistant Professor \\ Xian Aeronautical University, Xian, Shaanxi Province, China.
}

\begin{abstract}
The stable flight attitude movements of the quad-rotor UAV attained by using Fuzzy PID control algorithm in order to minimize the problems related to slow response and poor robustness. Firstly, the mathematical model of the Plus quad-rotor UAV model is established then the controller is designed which consist of inner ring angular velocity Fuzzy PID and outer ring angle P controller. The traditional techniques of the PID controller, cascade Fuzzy PID controller and cascade PID controller were simulated using Matlab/ Simulink to compare the step signal. The Fuzzy PID controller accelerates the results from the simulations show that the performance of the system improved in terms of accuracy of the system. The simulated flight result also shows that the Fuzzy PID controller shows robust behavior.
\end{abstract}

Keywords: Quad-rotor UAV, Fuzzy, PID, Attitude control.

\section{Introduction}

During the past few years, unmanned aerial vehicles (UAVs) are playing an important role in the field of aviation due to their novel structure, unique and exclusive flight mode and a wide range of use in different fields. As compared to the traditional fixed body structure aircrafts, UAV has simple mechanical structure with folding propellers operation. There are various interference factors, which are important for steady state flight response such as, turbulence due to strong winds. Some changes in the load weight and the algorithm of the UAV [1]. Presently, the control method of quad-rotor aircraft has linear flight control method, a model based nonlinear method and a learning based flight control method. Now it is clear that the PID controller reduces the traditional flight effect [2].

In this research article, the equations for the translation and rotation motion of the UAV are discussed and these equations are simplified to obtain the dynamic model of the quad-rotor. In the next, the design of cascade fuzzy PID controller for the aerial vehicle is consist on two PID controller in series to from a double closed loop PID controller as in the outer ring angle $\mathrm{P}$ controller and the inner ring is the fuzzy PID controller [3-6].

On the basis of Matlab/ Simulink simulations, the control system is compared and the simulation data is analyzed. Finally, the simulated results carried out, and the feasibility of the algorithm verified. 


\section{System Modeling and Preparation Knowledge}

In this research article, the quadcopter/quadrotor shown in the figure is considered as the research object. The two coordinate systems known as right-handed coordinate systems are developed which are machines and ground coordinate systems. Figure 1, shows the plus model of quadrotor aerial vehicle [7].

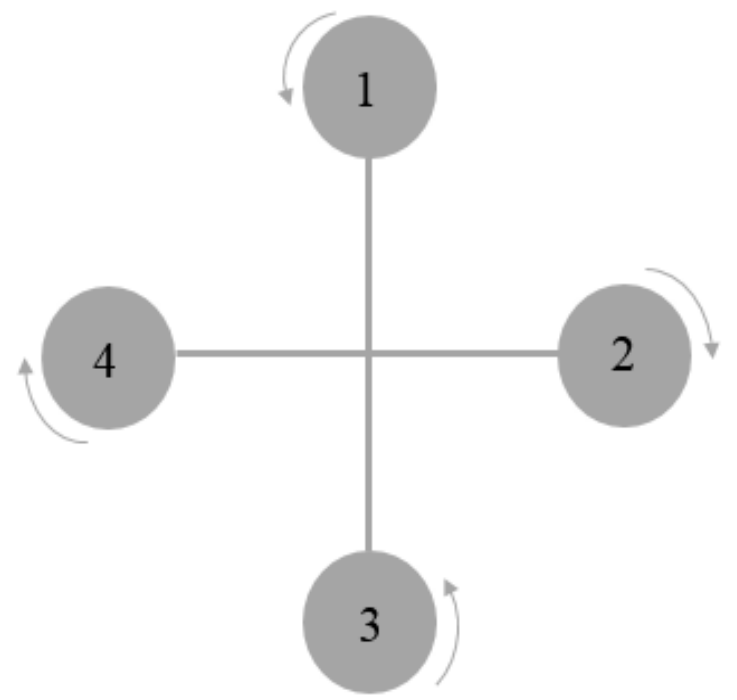

Figure 1: Plus shaped quadrotor

\section{Definition of related variables:}

- Center of gravity of UAV in relation with ground position variables in coordinate systems are $p=\left[\begin{array}{lll}x & y & Z\end{array}\right]^{T}$.

- Center of gravity of UAV in relation with ground velocity variables in coordinate systems are $v=\left[\begin{array}{lll}v_{x} & v_{y} & v_{z}\end{array}\right]^{T}$.

- UAV in relation with ground attitude variables in the plane coordinate systems are $\vartheta=$ $\left[\begin{array}{lll}\varphi & \theta & \Psi\end{array}\right]^{T}$.

UAV in relation with rotational velocity in the body coordinate systems are $\omega=\left[\begin{array}{lll}\omega_{x} & \omega_{y} & \omega_{z}\end{array}\right]^{T}$. The subscript $b$ and $d$ signifies the volume of the body and ground coordinate system. The transformation of vectors among the two-coordinate system needs rotation matrix [8]. The rotation matrix $R_{b}^{d}$ form body coordinate system to ground coordinate system is taken from [9] and rewritten as,

$$
R_{b}^{d}=\left[\begin{array}{ccc}
\cos \theta \cos \psi & \cos \psi \sin \theta \sin \varphi-\sin \psi \cos \theta & \cos \psi \sin \theta \cos \varphi+\sin \psi \sin \varphi \\
\cos \theta \sin \psi & \sin \psi \sin \theta \sin \varphi+\cos \psi \cos \varphi & \sin \psi \sin \theta \cos \varphi-\cos \psi \sin \varphi \\
-\sin \theta & \cos \theta \sin \varphi & \cos \cos \varphi
\end{array}\right]
$$




\subsection{System modeling}

With the structural symmetry, the quadrotor is assumed as a rigid body. The problems like gyro effect or air drag can easily be solved by Newton-Euler method [10]. The dynamic model of quadrotor UAV is shown as:

$$
\begin{gathered}
\dot{p}=v \\
\mathrm{~m} \dot{\mathrm{v}}=\mathrm{G}+\mathrm{T} \\
\dot{\Theta}=\mathrm{W} \times \omega \\
\mathrm{J} \cdot \dot{\omega}=-\omega \times(\mathrm{J} . \omega)+\tau
\end{gathered}
$$

Where $m$ is the mass of the quadrotor, $G$ and $\mathrm{T}$ act on the fly of drone respectively. Gravity on the device is given by $=\left[\begin{array}{lll}0 & 0 & \mathrm{mg}\end{array}\right]^{T}$; the force vector produced by the rotation of four thrusters is in the negative direction of the body coordinate system along the $O_{b} z_{b}$ axis is given by $f=$ $k_{T}\left(\Omega_{1}^{2}+\Omega_{2}^{2}+\Omega_{3}^{2}+\Omega_{4}^{2}\right)$ where $k$ is the lift coefficient, $\Omega_{i}=(i=1,2,3,4)$ speed of each rotor. The lift in the body coordinate system is changed into ground coordinate system $T=R_{b}^{d}\left(-f e_{3}\right)$, where $e_{3}=\left[\begin{array}{lll}0 & 0 & 1\end{array}\right]^{T}$ [11]. The center of mass translation models of UAV is given in equation (2) and (3). Equation (3) is expand to,

$$
\left.\begin{array}{l}
\dot{v}_{x}=-\frac{f}{m}(\cos \psi \sin \theta \cos \varphi+\sin \psi \sin \varphi) \\
\dot{v}_{y}=-\frac{f}{m}(\sin \psi \sin \theta \cos \varphi-\cos \psi \sin \varphi) \\
\dot{v}_{z}=g-\frac{f}{m}(\cos \theta \cos \varphi)
\end{array}\right\}
$$

The equation (4) and (5) describe the model of quadrotor UAV rotating about the center of mass. $W$ is the moment among the attitude rate and the angular rate of the body. $J$ is the moment of inertia matrix of the body coordinate system. The rotor of quadcopter is considered as a centrosymmetric object which can be written as $J=\left(J_{x}, J_{y}, J_{z}\right)$. The torque acting on the quadrotor is generated by gyroscopic effect. The gyroscopic effect includes drag torque, gyroscopic moment and aerodynamic force. In this research article, drag force and gyro moment are ignored. Roll, pitch and yaw moments are called aerodynamic moments. The results illustrate that the rolling torque is produced by the different speed of rotors. The different speed of rotors produces different pitching moments. The force produces anti-torque forces differing to the direction of rotation because of the air resistance [12]. The opposite torque of each rotor is unstable to generate yaw torque. The aerodynamic moments on the quadcopter are stated as;

$$
\tau=\left[\begin{array}{c}
\tau_{x} \\
\tau_{y} \\
\tau_{z}
\end{array}\right]=\left[\begin{array}{c}
d k_{T}\left(-\Omega_{2}^{2}+\Omega_{4}^{2}\right) \\
d k_{T}\left(\Omega_{1}^{2}-\Omega_{3}^{2}\right) \\
k_{Q}\left(\Omega_{1}^{2}-\Omega_{2}^{2}+\Omega_{3}^{2}-\Omega_{4}^{2}\right)
\end{array}\right]
$$

Where $d$ is the distance between the rotor and the body center, $k_{T}$ is the rotor lift coefficient and $k_{Q}$ is anti-torque coefficient. Expanding the formula (5) which gives 


$$
\left.\begin{array}{l}
\dot{\omega}_{x}=\frac{1}{J_{x}}\left[-\left(J_{z}-J_{y}\right) \omega_{y} \omega_{z}+\tau_{x}\right] \\
\dot{\omega}_{y}=\frac{1}{J_{y}}\left[-\left(J_{x}-J_{z}\right) \omega_{x} \omega_{z}+\tau_{y}\right] \\
\dot{\omega}_{z}=\frac{1}{J_{z}}\left[-\left(J_{y}-J_{x}\right) \omega_{x} \omega_{y}+\tau_{z}\right]
\end{array}\right\}
$$

\section{Fuzzy PID controller design}

\subsection{Fuzzy PID control principle}

As compared with the traditional PID controller, this article adopts Fuzzy PID controller. Outer loop is the angle control loop and inner loop is the angular velocity control loop because there is direct coupling between the motor and inner loop. The outer loop using the $\mathrm{P}$ controller in order to meet the stability requirements of the aircraft attitude control. In this research paper the roll angle, pitch angle and yaw angle control are discussed in fixed- height mode. The overall design of the controller is shown in figure 2.

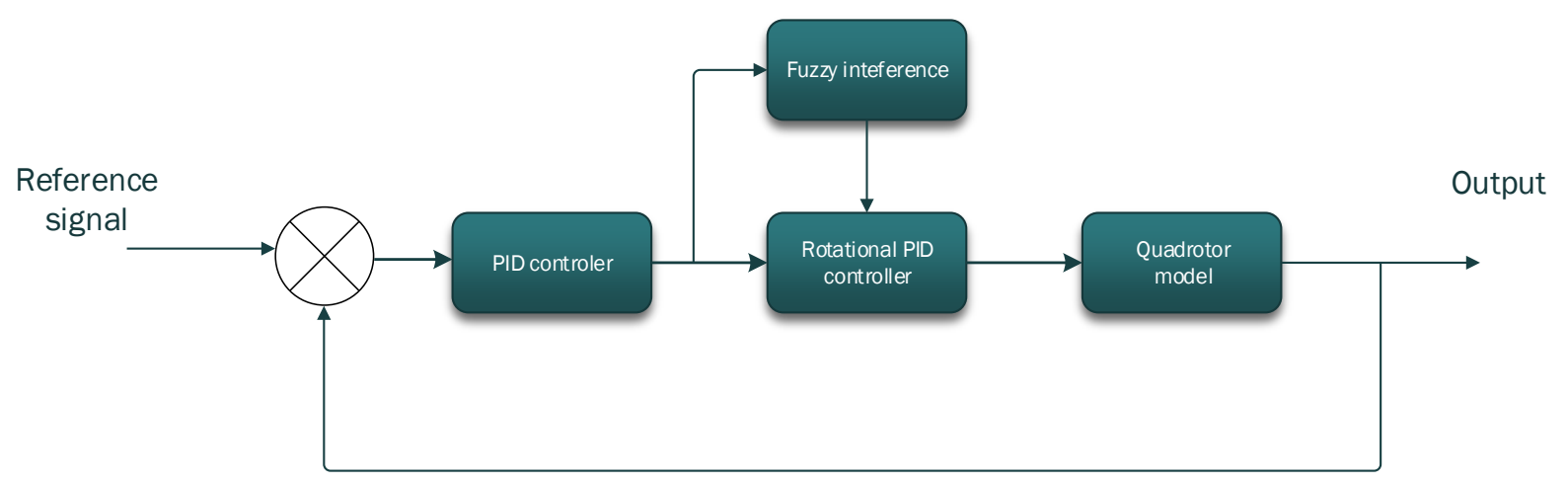

Figure 2: Controller block diagram

\subsection{External loop PID controller}

Firstly, it is expected that the aircraft will eventually be able to stabilize the flight to the desired target position, and the pitch angle will be independent during the centering process, assuming that the target trajectory is $R_{T}(t)$ and the final pitch angle is $\theta(t)$. Therefore, it is assumed that $R_{T}(t)=$ $R_{0}, \theta(t)=\theta_{0}$. The angular error is defined as:

$$
e_{\theta}=\theta_{r}-\theta_{a}
$$

$\theta_{r}$ is the actual pitch angle and $\theta_{a}$ is the desired pitch angle. Consequently, in order to make the angle error converge, the design of the outer ring angle is $\mathrm{P}$ controller based while the output of the controller as the inner ring output:

$$
\omega_{a}=K_{p 1}\left(\theta_{r}-\theta_{a}\right)
$$


Here; $\omega_{a}$ is the outer ring angle of the output is desired pitch angular velocity and $K_{p 1}$ is defined as the scale factor of the outer ring.

\subsection{Inner loop Fuzzy PID controller}

Inner loop fuzzy PID controller is mainly composed of Fuzzy interface, knowledge base fuzzy reasoning, PID controller and clear interface. It is particularly important that Fuzzy interference uses the deviation and the deviation rate of change as Fuzzy input. In the process of controlling system operation, the error and the rate of change of error are continuously detected and the three parameters of the PID controller are adjusted according to the predesigned Fuzzy rules. The designed Fuzzy PID control laws are defined in equation (11).

Where,

$$
\dot{\omega}=p\left(\omega_{\theta_{a}}-\omega_{\theta_{r}}\right)+i \int\left(\omega_{\theta_{a}}-\omega_{\theta_{r}}\right)+d\left(\dot{\omega}_{\theta_{a}}-\dot{\omega}_{\theta_{r}}\right)
$$

$$
\begin{aligned}
p & =K_{P}+\Delta K_{P} \\
i & =K_{i}+\Delta K_{i} \\
d & =K_{d}+\Delta K_{d}
\end{aligned}
$$

$\omega_{\theta_{a}}$ is the actual pitch angular velocity, $\omega_{\theta_{r}}$ is the desired pitch angular velocity, $\dot{\omega}$ is the output of inner fuzzy PID controller. $p, q, r$ are the angular velocities proportional coefficients of the inner PID controller after tuning, integration, differential coefficients which $\left(K_{P}, K_{i}, K_{d}\right)$ for the initial design value of PID parameters, $\left(\Delta K_{P}, \Delta K_{i}, \Delta K_{d}\right)$ for the Fuzzy controller.

\subsection{Fuzzy controller input and output variables and membership functions}

Two main parts of the angular velocity Fuzzy controller for Fuzzy reasoning and angular velocity PID controller are its error and its rate error and as a basis for real-time output PID controller parameter correction value. This paper is consist of three parameters of the PID controller $\left(\Delta K_{P}, \Delta K_{i}, \Delta K_{d}\right)$, since the three parameters of the PID controller is always in the dynamic adjustment, so the effect of fuzzy PID relative to the traditional PID control more significant.

In this research article, the attitude angle of the quad-rotor is altered in a small range, and the pitch angle is used as an example to carry out cascade Fuzzy PID control. In this respect, a clear amount of angular velocity deviation theory domain, a clear amount of angular acceleration, now take the angular velocity deviation rate of change of the quantization factor is quite less, therefore the Fuzzy angular velocity deviation of the domain is in between min and max range. The domain for Fuzzy angular acceleration deviation is also exist between min and max range. Since the Fuzzy interference section only changes the value of the output parameters, the initial value of the three parameters of PID is determined by Simulink simulations where the inner loop $\left(K_{P}=0.667, K_{i}=\right.$ $0.5, K_{d}=0.5$ ) the outer loop $K_{P}=0.49$, the inner loop fuzzy control $\Delta K_{P}, \Delta K_{i}, \Delta K_{d}$ domain is in the form of min and max values. By considering the response speed and control precision, the input and output variables are divided into grades, each grade corresponds to a Fuzzy set. To 
describe the Fuzzy variables, the Fuzzy subsets are $\{\mathrm{NB}, \mathrm{NS}, \mathrm{Z}, \mathrm{PS}, \mathrm{PB}\}$. In order to reduce the control quantity of the flight control system, both the input and output variables are selected with the Gaussian shape, the membership function of the middle triangle and the central method is used in the final defuzzification method.

TABLE 1: IF THEN FUZZY LOGIC RULE BASE FOR $K_{P}$

\begin{tabular}{|c|c|c|c|c|c|}
\hline ERROR & BN & SN & ZER & SP & BP \\
\hline$d e / d t$ & & & & & \\
\hline $\mathrm{BN}$ & ZER & SP & SP & SP & ZE \\
\hline SN & SP & SP & MP & PS & PS \\
\hline ZER & MP & SP & MP & MP & MP \\
\hline SP & MP & MP & MP & SP & LP \\
\hline BP & LP & MP & MP & SP & LP \\
\hline
\end{tabular}

TABLE 2: IF THEN FUZZY LOGIC RULE BASE FOR $K_{I}$

\begin{tabular}{|l|l|l|l|l|l|}
\hline ERROR & BN & SN & ZER & SP & BP \\
\hline$d e / d t$ & & & & & \\
\hline BN & ZER & ZER & ZER & ZER & ZER \\
\hline SN & ZER & SP & SP & SP & ZER \\
\hline ZER & SP & SP & SP & SP & SP \\
\hline SP & BP & SP & SP & SP & SP \\
\hline BP & BP & BP & BP & BP & BP \\
\hline
\end{tabular}

TABLE 3: IF THEN FUZZY LOGIC RULE BASE FOR $K_{D}$

\begin{tabular}{|l|l|l|l|l|l|}
\hline ERROR & BN & SN & ZER & SP & BP \\
\hline$d e / d t$ & & & & & \\
\hline NBN & SP & BP & BP & BP & BP \\
\hline NSN & SP & SP & SP & SP & BP \\
\hline ZER & ZER & SP & SP & SP & BP \\
\hline PSP & ZER & SP & SP & SP & SP \\
\hline PBP & ZER & BP & BP & BP & SP \\
\hline
\end{tabular}




\subsection{Fuzzy System rules}

Fuzzy PID control algorithm uses pre-speculated good Fuzzy rules to adjust the parameters, to the maximum time to meet the different requirements of self-tuning parameters, therefore the formation of reasonable Fuzzy rules for the attitude control of the UAV is extremely important. PID parameter Fuzzy rules are discussed in table 1 to 3 respectively. Fuzzy surface of the parameters is shown in figure 3 . In the fuzzy PID controller, $\Delta K_{p}$ is the scale factor, increasing the value of $\Delta K_{p}$ can make the actual angular velocity of the four-rotor aircraft closer to the desired angular velocity, so that the adjustment precision of the system is increased. For the aircraft, when the angular velocity error is large, it should increase the value $\Delta K_{p}$, so that the actual angle of the quad-rotor closer to the nearest desired attitude angle, with the reduction of errors it should the reduce the value of $\Delta K_{p}$ to avoid serious system overshoot, when the actual attitude angle become close to the desired attitude angle, approximately increases the value of to improve the static stability of the system. $\Delta k_{i}$ is integral coefficient, increasing $\Delta k_{i}$ can eliminate the steady-state error of the UAV. When the error is small, UAV is in steady state, can increase the value of $\Delta K_{i}$ which helps to improve the stability of the aircraft. When the error is large, the system is in the process of rapid response then if you increases the value of $\Delta k_{i}$ will make UAV integral saturation phenomenon, it is necessary to reduce the value of $\Delta k_{i}, \Delta k_{d}$ is the differential coefficient that can predict the amount of change in the system can be predicted in advance. When error is large, should increase the value of $\Delta k_{d}$ to inhibit the angular velocity of the aircraft overshoot, improve the dynamic performance of the system. When error is small, should reduce the value of $\Delta k_{d}$ otherwise the system adjustment time will be too large. The output rules of Fuzzy PID controller is seen in figure 4.

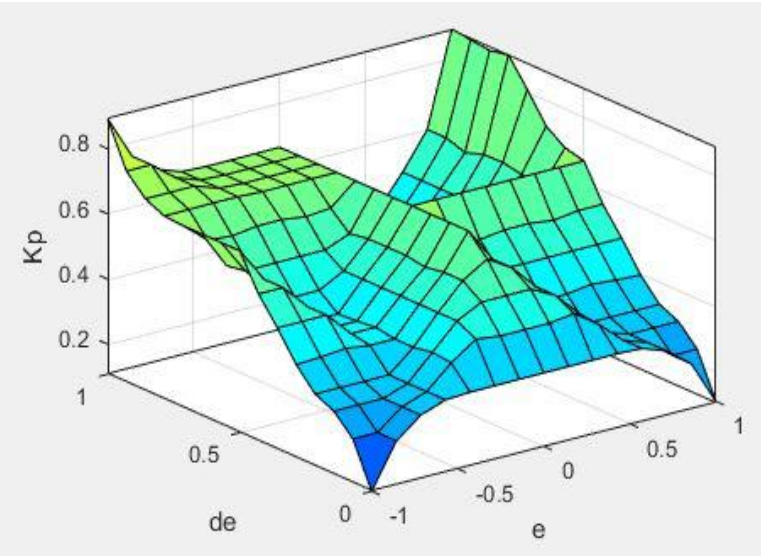

(a)

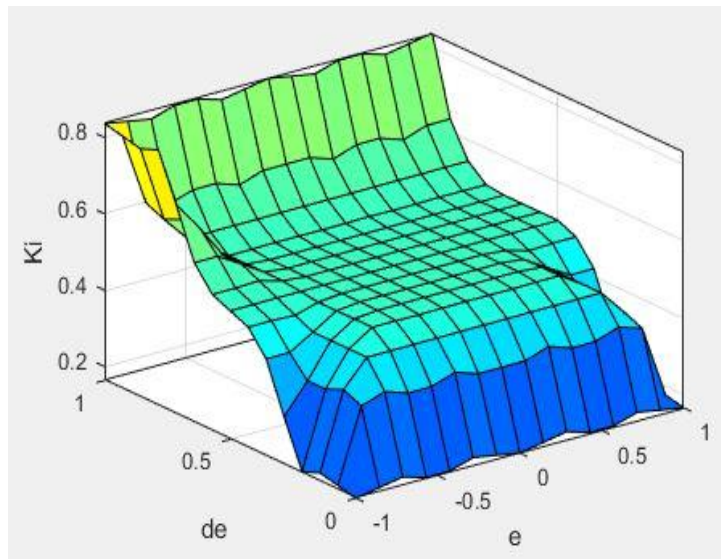

(b) 
Gyancity Journal of Engineering and Technology, Vol.6, No.1, pp. 1-11, January 2020

ISSN: 2456-0065 DOI: 10.21058/gjet.2020.61004

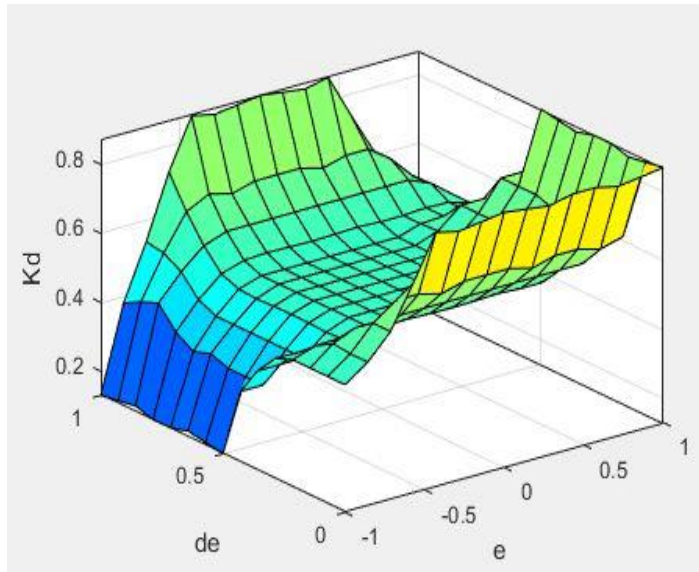

(c)

Figure 3 (a), (b) and (c): Fuzzy surface rules of PID controller

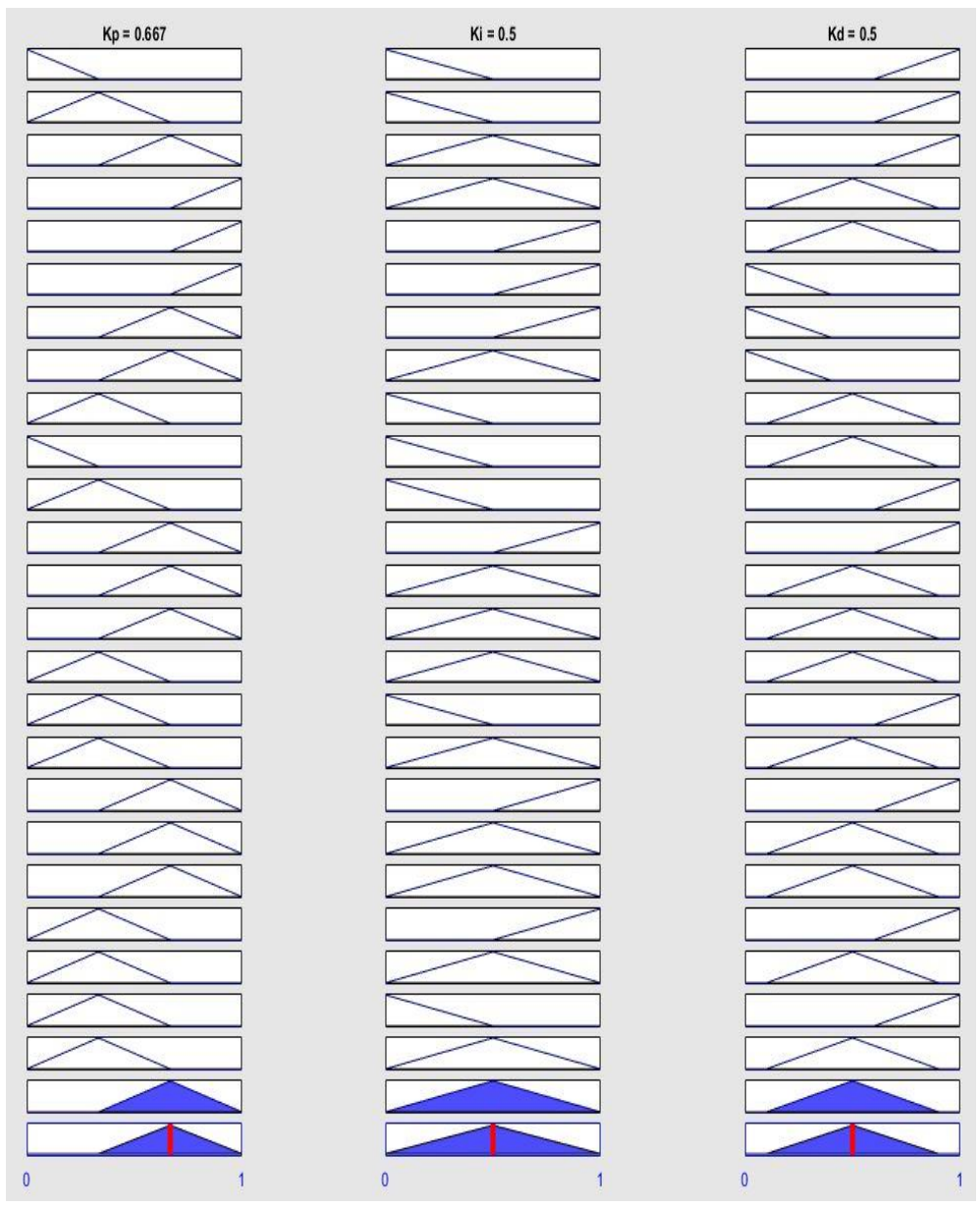

Figure 4: The output rules of Fuzzy PID controller 


\section{Simulation Results}

After designing the four-rotor control system, the simulation system parameters are defined in table 2. Matlab/ Simulink build simulations platform, the targeted angle control simulations of three methods, the simulations results shown in figure 5 and 6 respectively. Defines that the attitude angles are expected to step signal, Fuzzy PID control and level PID control, compared to traditional PID control, faster response time to each steady-state time, less overshoot, while the PID control to reach steady-state time quickly, with very overshoot. In order to verify the algorithm for PID parameters can be adjusted adaptively, given that when error and rate of error both are zero, the specific adjustment value of PID parameters.

Table 4: The simulation system parameters

\begin{tabular}{|c|c|}
\hline Parameters & Value \\
\hline $\mathrm{m} / \mathrm{kg}$ & 1.852 \\
\hline$g /\left(\mathrm{m} * \mathrm{~s}^{-2}\right)$ & 9.80 \\
\hline$l / m$ & 0.255 \\
\hline$I_{x} /\left(\mathrm{kg} * \mathrm{~m}^{-2}\right)$ & $9.313 \times 10^{-3}$ \\
\hline$I_{y} /\left(\mathrm{kg} * \mathrm{~m}^{-2}\right)$ & $9.313 \times 10^{-3}$ \\
\hline$I_{z} /\left(\mathrm{kg} * \mathrm{~m}^{-2}\right)$ & $1.672 \times 10^{-2}$ \\
\hline
\end{tabular}

In order to verify the effects of Fuzzy PID control algorithm on attitude robustness control of fourrotor aircrafts, this paper uses the plus frame of quadrotor as the carrier. Figure 5, shows the altitude approaching of aircraft which is commonly called as hovering mode, when UAV is stabilized at 1 meter height after that there attitude angles goes to the zero it means that there is no velocity at that time and UAV is still at same position. In this case, the roll, pitch and yaw moments are nearly or equal to zero which is shown in figure 6. 


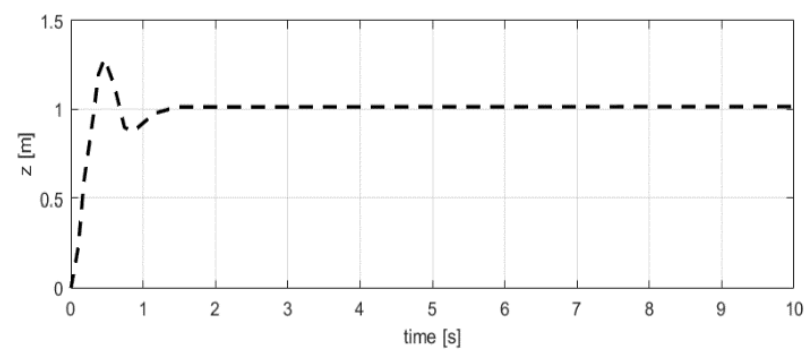

Figure 5: Altitude of quadrotor UAV

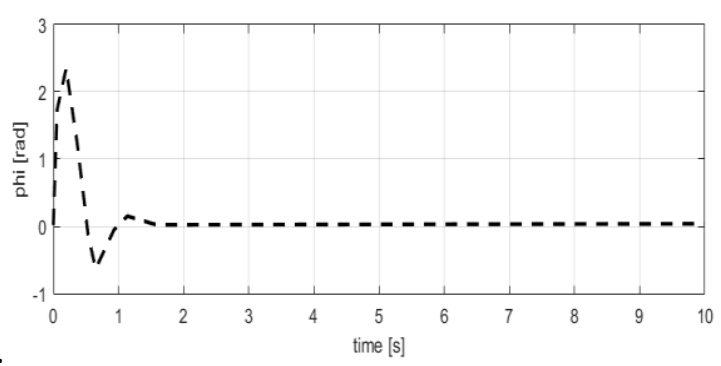

(a)

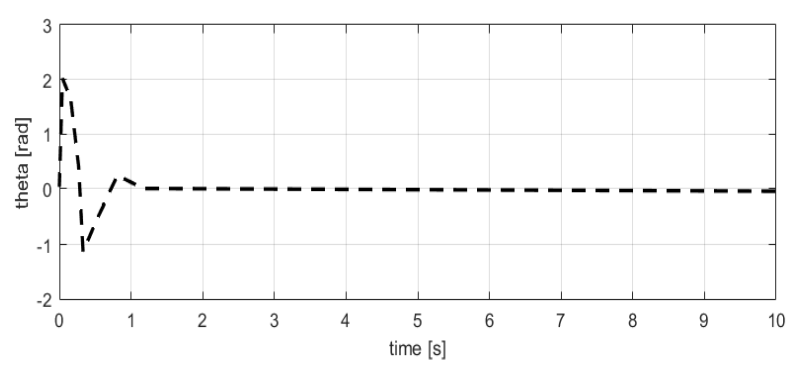

(b)

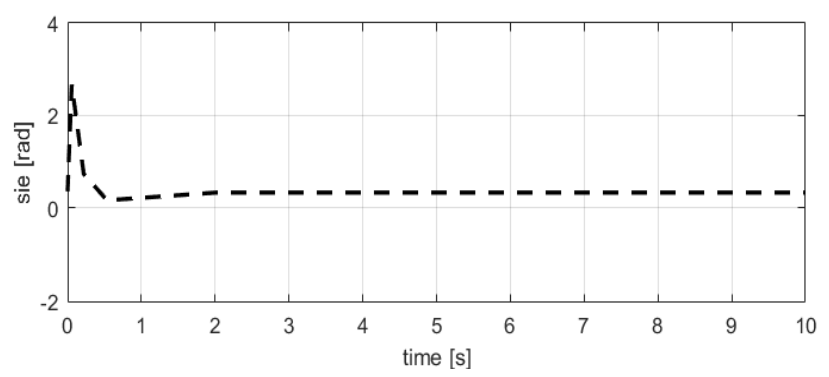

(c)

Figure $6(a, b, c)$ : The Euler or attitude angles of UAV

\section{Conclusion}

On the basis of attitude control of the UAV controller, the modeling analysis of the quad-rotor, the Fuzzy PID control algorithm is proposed, and the aircraft platform is setup in Matlab/ Simulink. The simulations results show that the Fuzzy PID control algorithm has high precision and fast response speed. In this research article, the angular velocity loop is used for Fuzzy control to control the angular rates of UAV and the cycle of attitude control is shortened, and thee dynamic characteristics of the attitude control are improved. 


\section{References}

1. Laihong Zhou, Juqian Zhang, Houxin She, \& Hong Jin. (2019). Quadrotor uav flight control via a novel saturation integral backstepping controller. Automatika, 60(2), 193-206.

2. Bristeau, P. J., Martin, P., Salaün, E., \& Petit, N. (2010). The Role of Propeller Aerodynamics in the Model of a Quadrotor UAV.

3. L.-H. Zhou, J.-X. Dou, J.-Q. Zhang, \& B.-C. Wen. (2018). Trajectory tracking control for a quadrotor uav based on improved backstepping. Dongbei Daxue Xuebao/journal of Northeastern University, 39(1), 66-70.

4. Tao, Y., Wang, W., Qiu, Y., \& Jie, L. I. (2018). Trajectory tracking control based on adaptive sliding mode for quadrotor uav.

5. Shuai An, Suozhong Yuan, \& Huadong Li. (2018). Adaptive trajectory tracking controller design for a quadrotor uav with payload variation. International Journal of Intelligent Computing and Cybernetics, 11(2).

6. Claudio Rosales, Carlos Soria, Ricardo Carelli, \& Francisco Rossomando. (2017). Adaptive dynamic control of a quadrotor for trajectory tracking. 2017 International Conference on Unmanned Aircraft Systems (ICUAS). IEEE.

7. Chuangchuang Zhu, Xiaolong Liang, Lvlong He, \& Liu Liu. (2017). Demonstration and verification system for UAV formation control. 2017 3rd IEEE International Conference on Control Science and Systems Engineering (ICCSSE). IEEE.

8. Fahmizal, Atikah Surriani, Ma'un Budiyanto, \& Muhammad Arrofiq. (2017). Altitude control of quadrotor using fuzzy self tuning PID controller. 2017 5th International Conference on Instrumentation, Control, and Automation (ICA). IEEE.

9. Allen Babb, \& Muhittin Yilmaz. (2017). Student research highlights: quadrotor electronics and intelligent control. IEEE Aerospace \& Electronic Systems Magazine, 32(6), 61-64.

10. Egidio D'Amato, Massimiliano Mattei, Agostino Mele, Immacolata Notaro, \& Valerio Scordamaglia. (2017). Fault tolerant low cost IMUS for UAVs. 2017 IEEE International Workshop on Measurements \& Networking (M\&N). IEEE.

11. Anurag Sai Vempati, Igor Gilitschenski, Juan Nieto, Paul Beardsley, \& Roland Siegwart. (2017). Onboard real-time dense reconstruction of large-scale environments for UAV. 2017 IEEE/RSJ International Conference on Intelligent Robots and Systems (IROS). IEEE.

12. Hahn, Wolfram, Engelke, Benjamin, Jung, Klaus, Dathe, Henning, Fialka-Fricke, Julia, \& KubeinMeesenburg, Dietmar等. . Initial forces and moments delivered by removable thermoplastic appliances during rotation of an upper central incisor. Angle Orthodontist, 80(2), 239-246. 